\title{
Impairment of notch 1 signaling is a common defect in lesions from patients with hidradenitis suppurativa
}

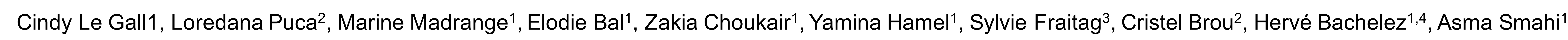

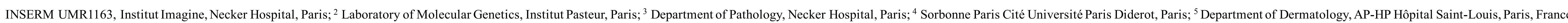

Introduction:

Hidradenitis suppurativa (HS), also called acne inversa (AI) (OMIM142690), is a chronic inflammatory disease charaterised by 2 major phenomenons: abnormalities of pilosebaceous follicular structures homeostasis, and an afflux of immune cells including macrophages, neutrophils and T lymphocytes. Although the pathogenesis of HS remains poorly understood to date, several lines of evidence support a strong genetic background interacting with environmental factors. Recently, a major advance was thet identification of causal genetic abnormalities in patients with severe HS, consting of mutations in genes coding for protein subunits the gamma-secretase (GS) complex, composed of four membrane proteins, presenilin (PS) constituting the catalytic compound, and three other subunits acting as necessary cofactors, Nicastrin (NCT), Anterior Pharynx Defective-1 (APH1), Presenilin Enhancer-2 (PSENEN). The GS complex mediates intramembranous cleavage of various type 1 membrane proteins including amyloid precursor and notch receptors (Bergmans b.a., Lancet Neurol, 2010). Notch signalling is initiated by ligand-receptor interaction between neighbouring cells (delta-like 1,3,4, jagged 1,2), In respons to ligand binding, notch proteins are cleaved by two proteases : a metalloprotease sheds the extracellular portion of the notch receptor. Subsequent cleavage by GS dissociates the notch intracellular domain (NICD) from the membrane which enters the nucleus, where it directly interacts with the RNA binding protein RBPjk leading to transcription of target genes (Mumm and Kopan, 2000, Lubman et aL, 2004). Notch molecules are expressed in the skin and the hair follicles (Favier et all, 1998) and have a complex role in controlling epidermal homeostasis (Powell et a, 2000; Kopan and Weintraub, 1993; Lin and Kopan, 2003; Yamamoto et al). In the present work, bearing in mind the well established role of GS subunits in the homeostasis of the notch pathway, we investigated first a large family where the HS trait results from a new mutation in the NCSTN gene. We addressed the genetic mechanisms and analyzed the impact of the nicastrin mutation on the notch signaling pathway, and investigated eventual impairment of the notch pathway at the tissular level in a series of patients with hidradenitis suppurativa (HS)/Al. Figure2: Immunohistochemical analysis of Notch 1 expression in perilesional skin
keratinocytes (B) and hair follicicle cells from a nodule (C,D) in patient III.4 reveals a predominant membrane pattern, strikingly different from

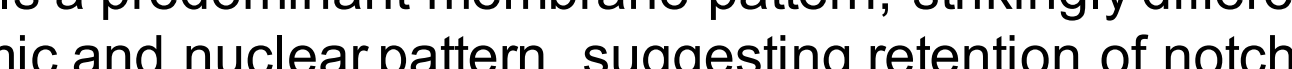

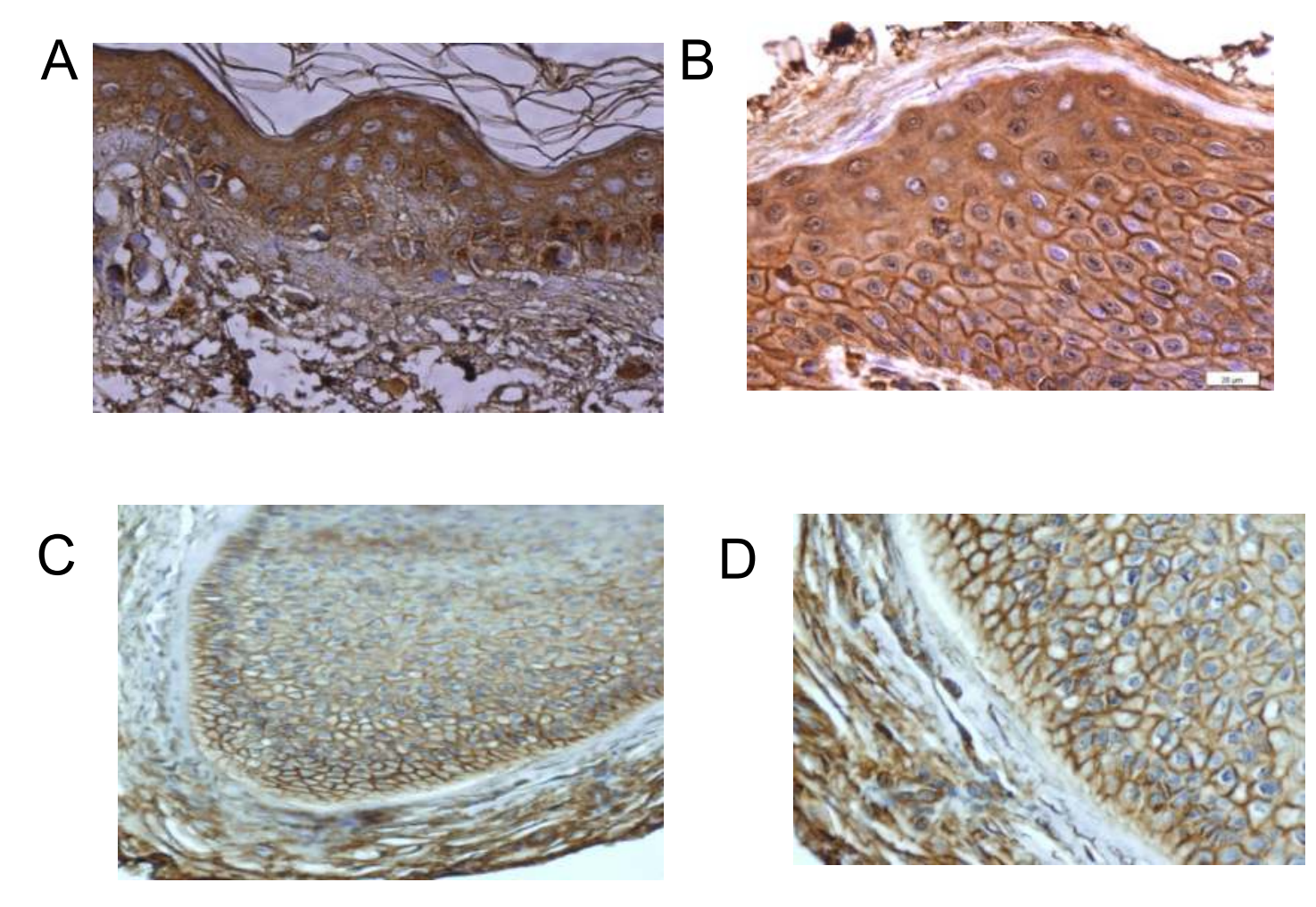

Figure 3: quantitative RT-PCR the transcriptional expression of 7 notch1-induced genes, CRABP2, DELTA1, HES1, JAG1, JAG2, NOTCH2, HEY epidermal splits from the 5 patients carrying the CSTNC1381 del C mutation, and in clinically nomal skin samples from 4 controls (A). A marked decrease
of all notch-1 driven genes transscription is observed. Similar downregulation is observed in lesions from 5 patients with Hurley grade III HS showing no

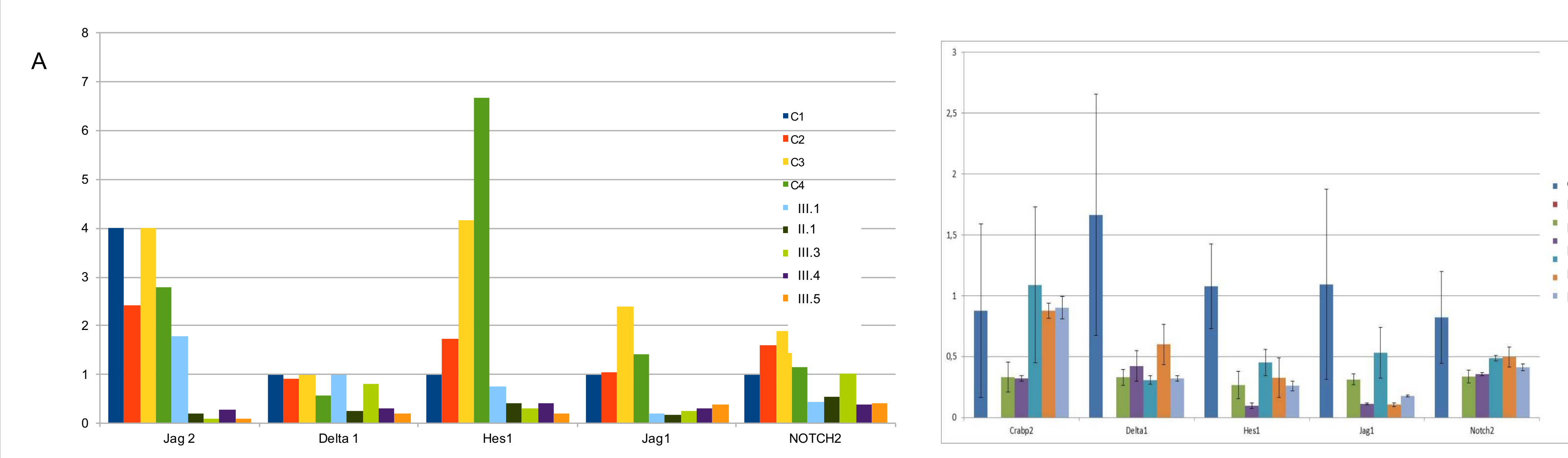

Figure3. In order to examine whether the mutant form of Nct could be expressed and affect g-secretase activity, we engineered expression vectors encoding
Nct with the same mutation that had been dettected in our patients, VSV-tagged or not (respectively named Nct mut-VSV and Nct mut). We transfected HEK resembling ADAM10 cleavage product that is generated after ligand activation (see Moretti et al 2010). Gamma-secretaseaccts directly on Notch DE and

Patients and Methods:

We performed whole genome linkage analysis using SNP 6.0 array on affected members from a large french family in which HS segregates as an autosomal dominant condition (figure 1) All affected individuals presented with Hurley grade 2 or 3 disease, with axillary and/or inguinal nodules, with facial and dorsal involvement in several cases. Three candidates regions with a maximum lod score of 2 were obtained. The 1q22-q23 region (genetic interval: 40928334158579687) includes the strong candidate gene NCSTN. Likewise, Sequencing of the whole NCSTN gene revealed a previously undescribed heterozygous cytosine insertion in exon11 (NCSTNC1381del C) predicted to result in a frame shift generating a premature stop codon at amino-acid position 424. No mutation was identified in genes encoding the 3 other GS components. The mutation segregates with the disease with complete penetrance. The mutated allele transcripts were present in whole skin, cystic lesions, keratinocytes, monocytes and EBV-B-immortalized cell lines. However the transcriptional expression level of the mutated allele was notably and consistently less than the wild type one in all these cases.

\section{Results}

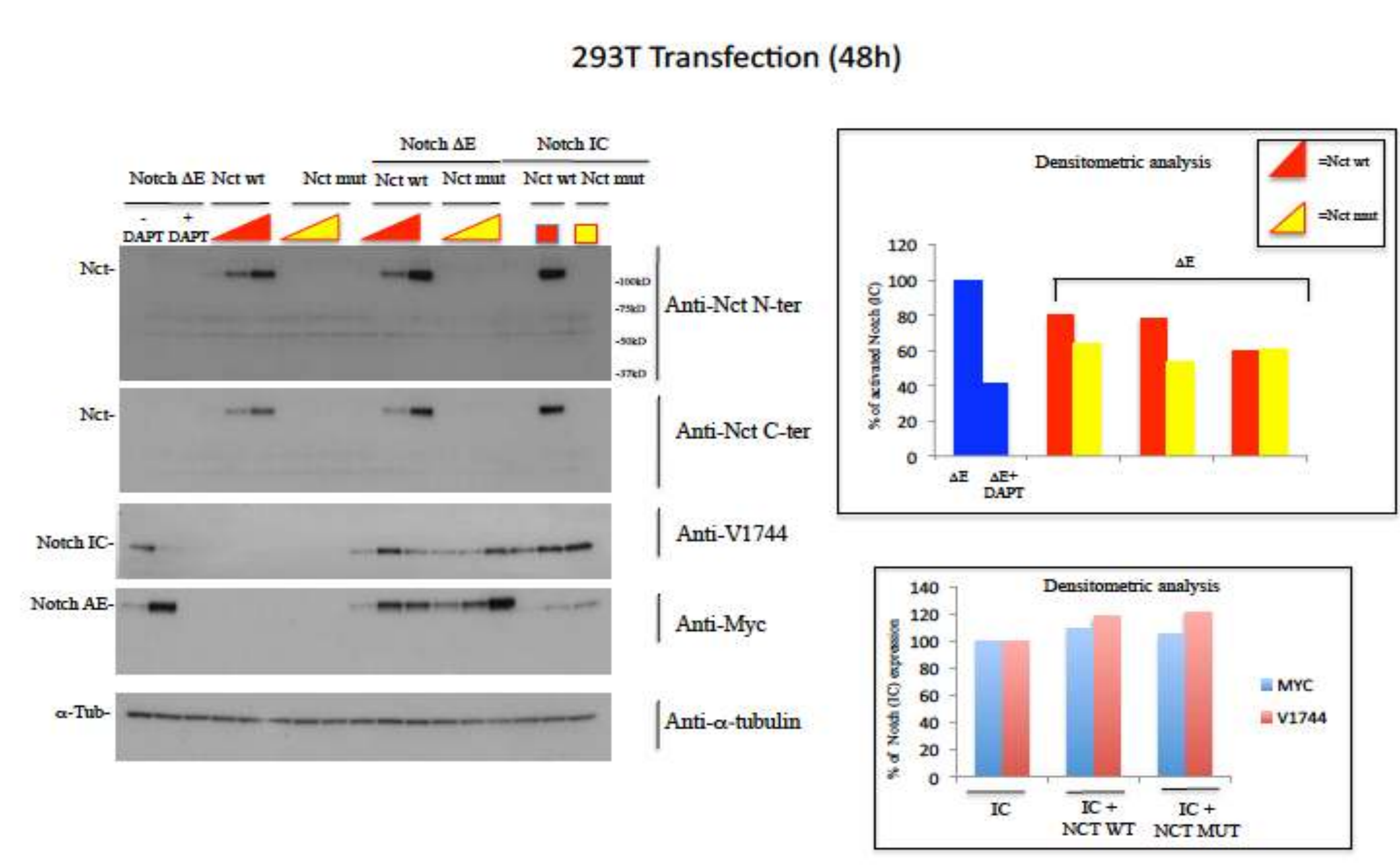

Figure 1: Mendelian segregation of the HS trait in a caucasian, french family. All affected individuals carry the same mutation of NCSTN, and all healthy individuals show no mutation in any gene of the GS complex.
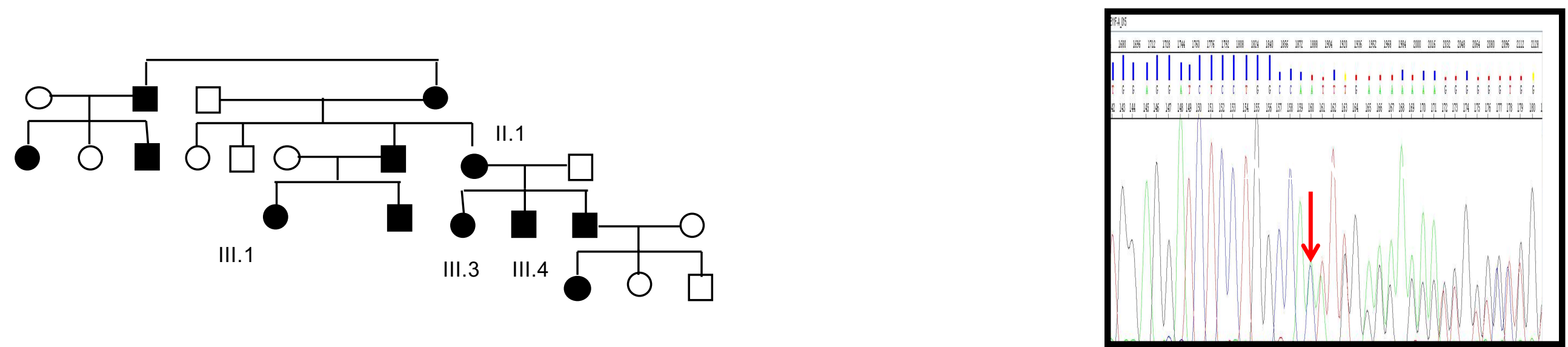

Conclusion: These results support that an ectopic expression of notch1 is shared across different genetic backgrounds in patients with HS, and that these abormalities lead to striking downregulation of notch-1 deoendent genes transcriptional expression, among which NOTCH2. These data are according to previous evidence that notch signalling and GS impaired function in mouse lead to abnormalities of skin appendages phenocopying those of HS (Pan Y, et al. Dev Cell 2004)

Taken together, these data also suggest that in patient cells, $\gamma$-secretase is still functional and that the NCSTNc1381delC mutation

does not act through a dominant negative effect. Previous studies showing that a reduction of nicastrin amount or of its activity leads to significant down regulation of notch-1 dependent genes and their encoded proteins in the skin provides additional evidence to this latter hypothesis (Li T, et al. J Biol Chem 2013). 